\title{
The effectiveness of adjustable oral appliance for older adult patients with obstructive sleep apnea syndrome
}

\author{
Rong-Jian Lu ${ }^{1 \# \wedge}$, Ning Tian ${ }^{2 \#}$, Jia-Zhu Wang ${ }^{3}$, Xuan Zou ${ }^{1}$, Jing-Jing Wang ${ }^{4}$, Min Zhang ${ }^{3}$, \\ Chang-Qing Bai ${ }^{4}$, Kai-Tao Yu ${ }^{1}$ \\ ${ }^{1}$ Department of Stomatology, ${ }^{2}$ Department of Radiology, Fifth Medical Center of Chinese PLA General Hospital, Beijing, China; ${ }^{3}$ Department of \\ Stomatology, First Medical Center of Chinese PLA General Hospital, Beijing, China; ${ }^{4}$ Department of Respiratory, Fifth Medical Center of Chinese \\ PLA General Hospital, Beijing, China \\ Contributions: (I) Conception and design: RJ Lu; (II) Administrative support: KT Yu, CQ Bai; (III) Provision of study materials or patients: N Tian, JJ \\ Wang; (IV) Collection and assembly of data: X Zou, M Zhang; (V) Data analysis and interpretation: X Zou; (VI) Manuscript writing: All authors; (VII) \\ Final approval of manuscript: All authors. \\ "These authors contributed equally to this work. \\ Correspondence to: Kai-Tao Yu. Department of Stomatology, Fifth Medical Center of Chinese PLA General Hospital, No. 8 Dongda Street, Fengtai \\ District, Beijing, China. Email: lujian9806@sohu.com; Chang-Qing Bai. Department of Respiratory, Fifth Medical Center of Chinese PLA General \\ Hospital, No. 8 Dongda Street, Fengtai District, Beijing, China. Email: 13426301158@163.com.
}

Background: Oral appliance (OA) treatment for obstructive sleep apnea syndrome (OSAS) has attracted more and more attention due to its low price, comfort, portable and non-invasion. This study aimed to investigate the clinical effectiveness of adjustable oral appliance on older adult patients with OSAS.

Methods: Thirty older adult patients diagnosed with OSAS were chosen as the study participants and received an adjustable OA for 6 months. Then, the patients were subjected to a polysomnographic examination, Berlin Questionnaire (BQ) scale questionnaire, and cone beam computer tomography (CBCT) analytical measurement to evaluate their symptom improvement and the morphologic changes of the upper airway.

Results: After treatment with adjustable oral appliance for six months, the results showed that there was an improvement of different degrees in the subjective symptoms. Apnea hypopnea index (AHI) had decreased from $(27.65 \pm 1.31)$ per hour to $(6.74 \pm 0.75)$ per hour $(\mathrm{P}<0.05)$; the maximum apnea time $($ MAT) had decreased from $43.82 \pm 2.69$ to $21.37 \pm 3.18 \mathrm{~s}(\mathrm{P}<0.05)$; the average oxygen saturation $\left(\mathrm{MSaO}_{2}\right)$ had increased from $(89.24 \pm 7.27) \%$ to $(92.69 \pm 4.46) \%$; the lowest oxygen saturation $\left(\mathrm{LSaO}_{2}\right)$ from $(81.85 \pm 8.31) \%$ to $(86.93 \pm 4.45) \%$. Moreover, the CBCT scanning analysis showed that the minimal sagittal diameter, sectional area, and the volume of the palatopharynx, as well as the sagittal diameter and volume of the glossopharynx significantly increased.

Conclusions: The adjustable OA had considerable clinical efficacy and comfort in older adult OSAS patients by enlarging the palatopharynx and glossopharynx.

Keywords: Adjustable oral appliance; obstructive sleep apnea syndrome (OSAS); older adults; palatopharynx; glossopharynx

Submitted May 25, 2020. Accepted for publication Jul 10, 2020.

doi: 10.21037/apm-20-1300

View this article at: http://dx.doi.org/10.21037/apm-20-1300

^ ORCID: 0000-0002-0585-9370. 


\section{Introduction}

Obstructive sleep apnea syndrome (OSAS) is a common sleep-disordered breathing disease characterized by recurrent apnea or hypopnea during sleep. Its clinical manifestations include intermittent snoring at night, conscious suffocation, daytime sleepiness, inattention and emotional irritability. Disrupted sleep can result in hypersomnolence and impaired concentration, increased probability of traffic accidents, and decreased quality of life. OSAS is also associated with adverse clinical outcomes, including cardiovascular disease, hypertension, diabetes and an increased risk for respiratory complications $(1,2)$.

The prevalence of OSAS is $3 \%$ to $7 \%$, while the prevalence of OSAS in the older adults' population is as high as $40 \%$. The proportion of the older adult population in China with OSAS is high, and as the population ages, the number of older adult OSAS patients continues to rise. The OSAS in older adults has a prolonged course of the disease, and its damage to various system organs is severe. The disease has also become a public health problem that jeopardizes the health of the older adults, and its early prevention and treatment have attracted increasing attention.

Continuous positive airway pressure (CPAP) is a most reliable treatment for OSAS in addition to weight loss and alcohol avoidance, unfortunately many patients reject CPAP or tolerate it only partially. And the elderly are not suitable for surgical procedures such as glossectomy and uvulopalatopharyngoplasty. As a means of the conservative treatment of OSAS, the adjustable oral appliance (OA) has the advantages of safety, effectiveness, simplicity, portability, and low cost. It achieves the purpose of expanding or stabilizing the upper airway by changing the positional relationship between the jaw, tongue, soft palate, and palate. Simultaneously, by adjusting the positioning of the jaw, the treatment achieves a balance between a satisfactory curative effect and mandibular comfort (3).

The efficacy of the OA for the treatment of children and adults with OSAS has been established. There is considerable evidence that aging could be associated with changes in OSAS-related parameters and type of sleep. Moreover, the number of obstruction sites and the collapse pattern may change with aging. However, clinical evaluations on the $\mathrm{OA}$ for the treatment of elderly patients with OSAS have rarely been reported. Therefore, we conducted this study to investigate the clinical effectiveness of the adjustable OA in older adult patients with OSAS by examining the patients' subjective symptoms, the sleep breathing parameters of older adult patients, and the threedimensional morphological changes of the upper airway.

We present the following article in accordance with the TREND reporting checklist (available at http://dx.doi. org/10.21037/apm-20-1300).

\section{Methods}

\section{Subjects}

From September 2018 to September 2019, thirty older adult patients were diagnosed as OSAS by a polysomnography instrument (repeated episodes of apnea $\geq 30$ times or AHI $\geq 5$ times/hour during 7 hours of sleep) in the Department of Respiration of The Fifth Medical Center of the PLA General Hospital and enrolled in the present study. All procedures performed in this study involving human participants were in accordance with the Declaration of Helsinki (as revised in 2013). The study was approved by Ethics Committee of the 307 Hospital of PLA (No. ky2018-9-83). And all patients signed informed consent.

\section{Inclusion and exclusion criteria}

Inclusion criteria: (I) mild to moderate OSAS patients diagnosed according to the diagnosis and treatment guidelines; (II) patients that were older than 60 years old; (III) patients that had more than 12 residual teeth in a single occlusion.

Exclusion criteria: (I) patients with severe OSAS who need surgical treatment; (II) patients with severe dentition loss, residual teeth loosening higher than the degree of II or temporomandibular joint disorders; (III) patients that had airway obstruction caused by ear, nose, and throat diseases; (IV) patients that had other diseases affecting respiratory status, including chronic obstructive pulmonary disease; (V) patients that had or were receiving other OSAS-related treatments.

\section{The manufacture and wearing of the adjustable $O A$}

A personalized, adjustable OA was constructed according to the dentition model of OSAS patients. The transparent upper and lower jaw pad was constructed by a soft plastic pressing diaphragm at $155^{\circ} \mathrm{C}$ and its thickness was about $3 \mathrm{~mm}$, which was linked by a connecting rod (ERKODENT Germany). The connecting rod has different lengths from 21 to $26 \mathrm{~mm}$ and can be selected according to the occlusion condition of the patients (Figure 1). 

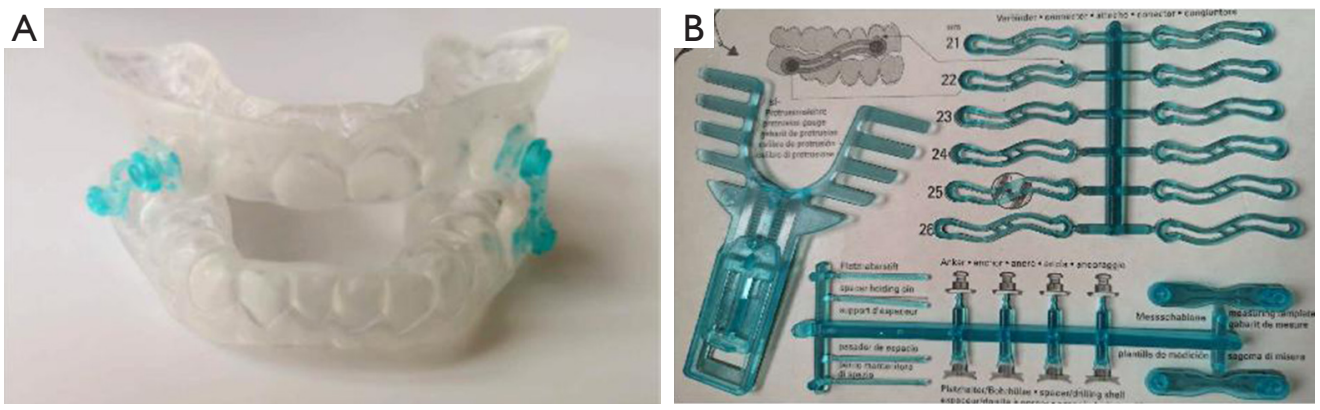

Figure 1 The constitution and fitting of the adjustable oral appliance. (A) Monolithic component; (B) fittings of the adjustable oral appliance.

The wax recording was taken at the maximum mandibular protrusion position of the OSAS patient, which was completed by experienced clinicians. At $65-75 \%$ of the maximum forward extension distance, the upper and lower plaster models were fixed to the maxillary frame. Until the temporomandibular joint was confirmed to have no discomforting symptoms, the connecting rod fixed the upper and lower jaw pads. The anterior tooth area of the appliance has an airway with an opening of $2-5 \mathrm{~mm}$ while supporting a certain degree of physiological mobility. Under the guidance of the doctor, the patients wore a personalized, adjustable OA nightly during sleep. After one week of the OA trial, the length of the connecting rod was adjusted according to the patient's complaint symptoms so that the patient's jaw was in a more comfortable position.

\section{Subjective symptoms}

The older adult OSAS patients completed a questionnaire under the direction of the researchers to investigate the improvement of the subjective symptoms in older adult OSAS patients treated with an adjustable appliance. The Berlin Questionnaire (BQ) score reflected the patients' snoring, sleepiness, and fatigue since they woke up in the morning.

\section{Polysomnography (PSG)}

A polysomnography monitor (EMBLA S4500, USA) was applied to observe the changes in oxygen supply during nighttime sleep after the patients received the OSAS treatment. The monitoring indexes include the apneahypopnea index (AHI), mean oxyhemoglobin saturation $\left(\mathrm{MSaO}_{2}\right)$, lowest $\mathrm{SaO}_{2}\left(\mathrm{LSaO}_{2}\right)$, and maximum apnea time (MAT). All operations and data analysis are performed by physicians in the respiratory sleep center.

\section{The CBCT examination of the upper airway morphology}

CBCT (KaVo 3DXam, Germany) was used to scan the patients' upper airway. The OSAS patient sat upright with his/her eyes looking (at the front and horizontally) straight ahead, the Frankfort Horizontal plane was parallel to the ground, and the patient's posterior teeth were naturally biting into the $\mathrm{OA}$ in the central jaw position without swallowing and while breathing quietly.

As shown in Figure 2, the measurement markers are PNS (posterior nasal spinous point), $\mathrm{U}$ (uvula apex point), and Ep (epiglottis apex point).

Palatopharyngeal airway: the upper boundary passes the PNS point and is parallel to the $\mathrm{FH}$, while the lower boundary passes the $\mathrm{U}$ point and is parallel to the $\mathrm{FH}$. Glossopharyngeal airway: the upper boundary is parallel to the $\mathrm{FH}$ that passes the $\mathrm{U}$ point, while the lower boundary is the plane that passes the Ep point and is parallel to the $\mathrm{FH}$ (Figure 3).

In this study, the palatopharyngeal and glossopharyngeal segment of the upper airway were taken as the investigation objects, and the measurement indexes were the sagittal diameter, coronal diameter, cross-sectional area, and volume of the minimum cross-section of the palatopharyngeal segment and the glossopharyngeal segment.

\section{Statistical analysis}

We used the software program SPSS 20.0 (IBM, Chicago, USA) to conduct the statistical analysis. Continuous variables were expressed as mean $\pm \mathrm{SD}$, and discontinuous variables were expressed as a percentage (\%). A value of $\mathrm{P}<0.05$ was considered statistically significant. 

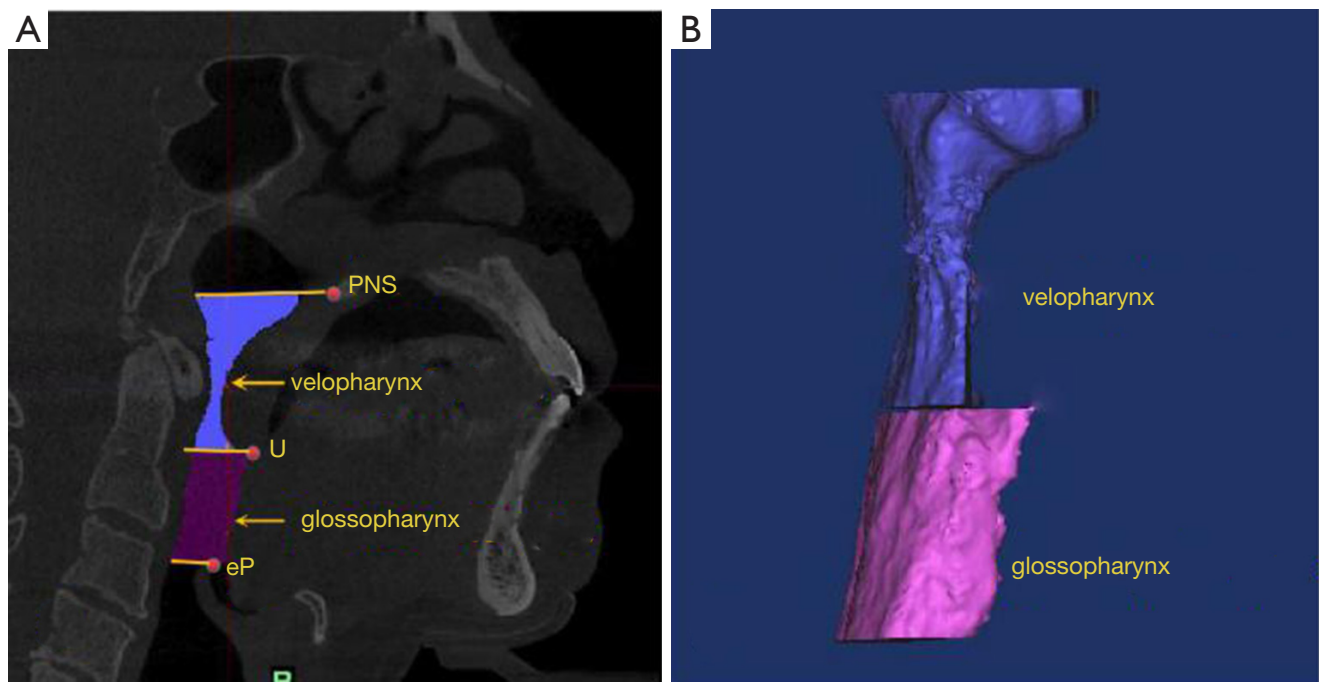

Figure 2 The boundary of velopharyngeal and glossopharyngeal airway. (A) The cephalometric landmarks and lines on the midsagittal plane; (B) the three-dimensional model of velopharyngeal and glossopharyngeal airway.
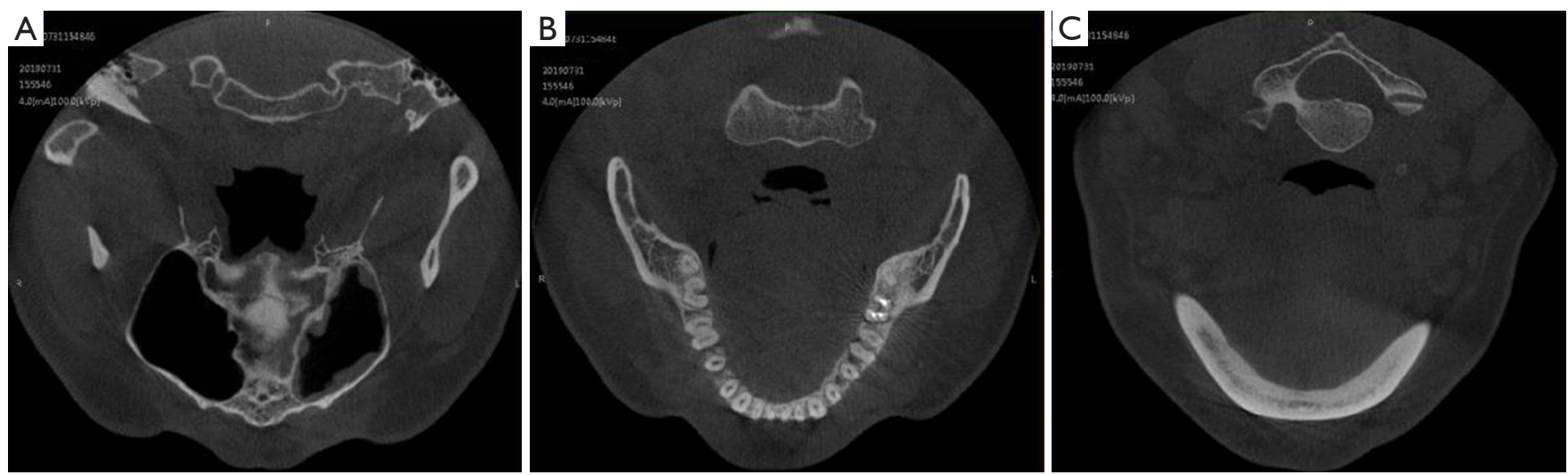

Figure 3 The boundary planes of oropharyngeal airway. (A) The upper boundary plane of velopharyngeal airway (PNS); (B) the lower boundary plane of velopharyngeal airway $(\mathrm{U}) ;(\mathrm{C})$ the lower boundary plane of the glossopharyngeal airway (Ep).

\section{Results}

\section{The general characteristics}

A total of 30 older adult patients (aged $60-70$ years) were included in this study. Among these patients, there were 22 males and 8 females. The mean body mass index (BMI) of the patients was $27.2 \pm 3.8$. According to the respiratory disorder index (AHI) and the lowest oxygen saturation $\left(\mathrm{LaSaO}_{2}\right)$, the patients were divided into 12 mild cases and 18 moderate cases.

\section{Improvement of the subjective symptoms}

After 6 months of treatment with the adjustable OA, the older adult patients showed the most obvious improvement in their snoring symptoms and a lower memory loss (Table 1). The BQ questionnaire showed that symptoms, including the snoring and morning drowsiness score, also significantly decreased $(\mathrm{P}<0.05)$ (Table 2$)$. Some patients initially had, uncomfortable symptoms, including complaining about temporomandibular joint discomfort, tooth pain, increased saliva secretion, and the looseness of 
Table 1 Subjective changes of elderly patients with OSAS treated by adjustable oral appliance after 6 months

\begin{tabular}{lccccc}
\hline Symptoms & Cases & Disappearance & Improvement & No improvement & Effective rate (\%) \\
\hline Snoring & 28 & 7 & 20 & 1 & 96.43 \\
Oppressive wake & 15 & 5 & 8 & 2 & 86.67 \\
Daytime sleepiness & 21 & 4 & 14 & 3 & 85.71 \\
Memory decline & 22 & 1 & 17 & 4 & 81.82 \\
\hline
\end{tabular}

OSAS, obstructive sleep apnea syndrome.

Table 2 BQ questionnaire score of older adult patients with OSAS treated by adjustable oral appliance after 6 months

\begin{tabular}{lccc}
\hline Symptoms & Before treatment & After treatment & P value \\
\hline Snoring & $2.87 \pm 0.95$ & $0.74 \pm 0.53$ & 0.012 \\
Morning fatigue and sleepiness & $2.49 \pm 0.74$ & $0.26 \pm 0.22$ & 0.019 \\
Total score & $2.68 \pm 0.49$ & $0.65 \pm 0.31$ & 0.033 \\
\hline
\end{tabular}

OSAS, obstructive sleep apnea syndrome.

Table 3 PSG changes of older adult patients with OSAS treated by adjustable oral appliance after 6 months

\begin{tabular}{lccc}
\hline Monitoring index & Before treatment & After treatment & P value \\
\hline $\mathrm{AHI}($ times/h) & $27.65 \pm 1.31$ & $6.74 \pm 0.75$ & 0.018 \\
$\mathrm{MSaO}_{2}(\%)$ & $89.24 \pm 7.27$ & $92.69 \pm 4.46$ & 0.039 \\
$\mathrm{LSaO}_{2}(\%)$ & $81.85 \pm 8.31$ & $86.93 \pm 4.55$ & 0.044 \\
$\mathrm{MAT}(\mathrm{s})$ & $43.82 \pm 2.69$ & $21.37 \pm 3.18$ & 0.027 \\
\hline
\end{tabular}

OSAS, obstructive sleep apnea syndrome; AHI, apnea hypopnea index; MAT, maximum apnea time.

their appliances. After two weeks of adjustment, the patients were satisfied with the orthodontics and had no noticeable discomfort.

\section{The analysis of the PSG results}

As shown in Table 3, after receiving treatment with the $\mathrm{OA}$, the AHI and the longest apnea time (MAT) of the older adult patients with OSAS respectively reduced from $27.65 \pm 1.31$ and $43.82 \pm 2.69$ to $6.74 \pm 0.75$ and $21.37 \pm 3.18$. Simultaneously, the average oxygen saturation $\left(\mathrm{MSaO}_{2}\right)$ and the lowest oxygen saturation $\left(\mathrm{LSaO}_{2}\right)$ increased from $89.24 \pm 7.27$ and $81.85 \pm 8.31$ to $92.69 \pm 4.46$ and $86.93 \pm 4.45$, and the difference was statistically significant $(\mathrm{P}<0.05)$.

\section{The CBCT measurement and analysis of the upper airway morphology}

After 6 months of treatment with the orthodontic apparatus, the sagittal diameter, sectional area, and volume of the smallest section of the palatopharyngeal segment in the older adult patients increased, and the difference was statistically significant $(\mathrm{P}<0.05)$. The sagittal diameter and volume of the pharyngeal segment of the tongue also increased significantly. However, there were no significant changes in the minimum palatopharyngeal diameter, the minimum palatopharyngeal diameter, or the minimum palatopharyngeal diameter, and the cross-section area (P>0.05) (Tables 4,5 and Figure 4). 
Table 4 CBCT measurement of velopharynx of older adult patients with OSAS treated by adjustable oral appliance

\begin{tabular}{lccc}
\hline Measurement index & Before treatment & After treatment & P value \\
\hline Minimum sagittal diameter $(\mathrm{mm})$ & $8.85 \pm 2.31$ & $10.76 \pm 2.53$ & 0.031 \\
Minimum transverse diameter $(\mathrm{mm})$ & $11.24 \pm 3.29$ & $17.82 \pm 4.78$ & 0.048 \\
Minimum section area $\left(\mathrm{mm}^{2}\right)$ & $203 \pm 30.76$ & $269 \pm 42.66$ & 0.019 \\
Minimum volume $\left(\mathrm{mm}^{3}\right)$ & $5,752 \pm 269.27$ & $8,579 \pm 306.57$ & 0.027 \\
\hline
\end{tabular}

OSAS, obstructive sleep apnea syndrome; CBCT, cone-beam computed tomography.

Table 5 CBCT measurement of glossopharynx of older adult patients with OSAS treated by adjustable oral appliance

\begin{tabular}{lccc}
\hline Measurement index & Before treatment & After treatment & P value \\
\hline Minimum sagittal diameter $(\mathrm{mm})$ & $7.08 \pm 2.58$ & $10.08 \pm 3.12$ & 0.026 \\
Minimum transverse diameter $(\mathrm{mm})$ & $10.14 \pm 2.95$ & $16.72 \pm 4.52$ & 0.072 \\
Minimum section area $\left(\mathrm{mm}^{2}\right)$ & $182 \pm 43.56$ & $258 \pm 32.63$ & 0.058 \\
Minimum volume $\left(\mathrm{mm}^{3}\right)$ & $6,547 \pm 347.38$ & $7,476 \pm 325.35$ & 0.033 \\
\hline
\end{tabular}

OSAS, obstructive sleep apnea syndrome; CBCT, cone-beam computed tomography.
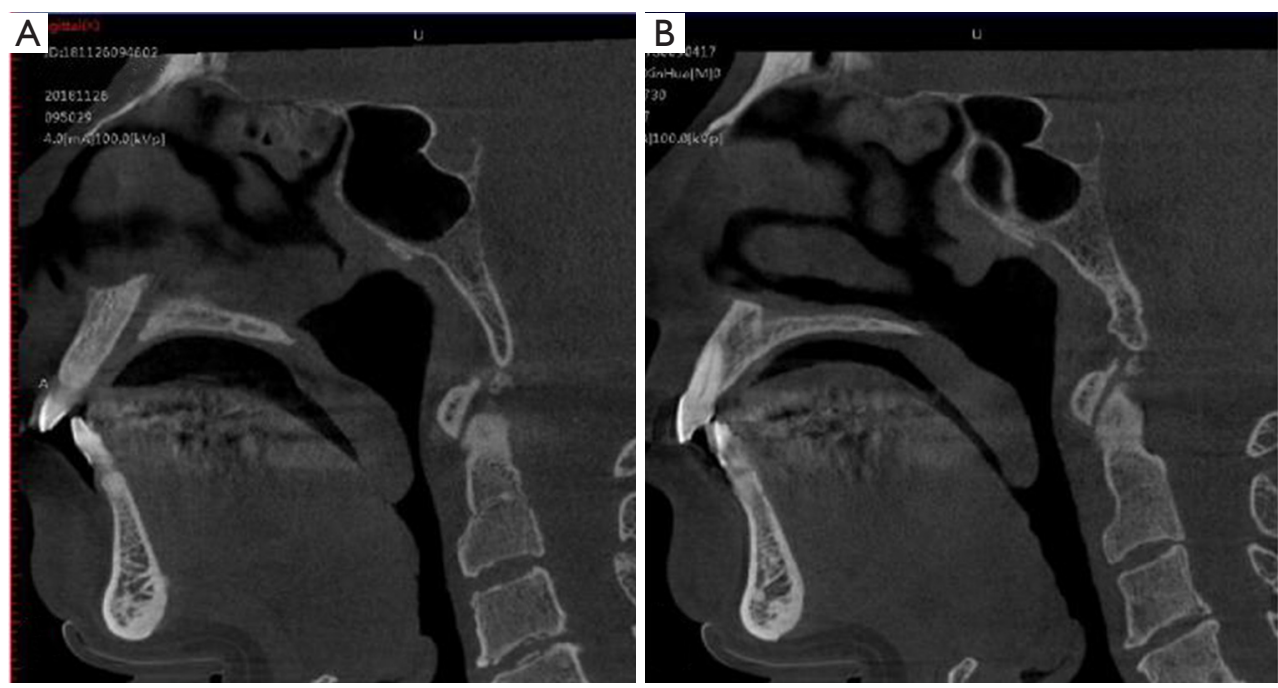

Figure 4 Morphological comparison of the upper airway of older adult patients with OSAS using CBCT. (A) Before treatment with adjustable oral appliance; (B) after treatment with adjustable oral appliance. OSAS, obstructive sleep apnea syndrome; CBCT, cone-beam computed tomography.

\section{Discussion}

OSAS refers to more than30 repeated seizures of apnea or $\mathrm{AHI} \geq 5$ times/hour during 7 hours of sleep nightly. Earlier studies have shown that old age and obesity are risk factors for OSAS (4). The prevalence of OSAS in the older adult population was much higher than that found in the middle and young population. There are nearly 250 million people over 60 years of age in China, accounting for $18 \%$ of the total population, and the proportion of older adult obese people are as high as $27.3 \%(5,6)$. The prevalence of OSAS in older adults showed a significant upward trend. The incidence of cardiovascular and cerebrovascular diseases in older adult patients with OSAS was significantly higher 
than that in young patients. It is reported (7) that OSAS is closely related to hypertension; more than $50 \%$ of OSAS patients also experience complications with hypertension, especially in obese and older adult patients.

After these OSAS patients were treated, their blood pressure had been significantly reduced or stabilized (8). Ifergane et al. (9) also discovered the prevalence of OSAS in stroke patients through the prolonged course of the disease, older OSAS patients have more severe nighttime hypoxemia, which can lead to hypoxemia, and hypercapnia and the damage to various organ functions are more severe than young people. OSAS is also an independent risk factor for hypertension, coronary heart disease, cerebral infarction, and diabetes, which seriously endangers the health of the older adult population. However, the symptoms of sleepiness and attention loss in older adult OSAS patients are easily confused with their physiological aging, leading to an overlooked diagnosis or misdiagnosis. As the first cause of the above complications, OSAS is also easily neglected. Therefore, early prevention and the timely and effective diagnosis and treatment of OSAS in the older adult population are necessary.

Nasal continuous positive airway pressure (CPAP) is considered an effective method for the management of OSAS, but the high cost is challenging, and the inconvenience of the long-term compliance of treatment in older adults with OSAS is less than $40 \%$. Moreover, the symptoms of OSAS in older adults are different from younger age groups, and there is not enough clinical evidence for the effectiveness of CPAP (10). Some psychologists also suggest that CPAP is not the preferred method for older adult OSAS patients. However, the success rate of high-risk surgical treatment is lower than $50 \%$. Because older adult OSAS patients are complicated with multiple system diseases, it is unsuitable for the initial treatment of senile OSAS (11).

The OA is an ideal alternative treatment for OSAS due to its simplicity, efficiency, and convenience, especially for patients with mild or moderate OSAS. The adjustable dental appliances used in this study can consider both significant clinical effects and comfort, more natural for patients to accept, and are more effective for long-term treatment. The objective effective rate was $88.2 \%$, and the effective subjective rate was $93.3 \%$ (12). Many clinical studies have confirmed that adjustable dental appliances can change mandibular positioning, effectively increase the airway cross-sectional area, and effectively reduce the respiratory disorder index in OSAS patients (13).
However, this method was not the preferred treatment before, and due to severe tooth loss in older adults in the past, the retention effect of the dental appliances was poor. With the improvement of medicine and the increased awareness of oral health, the number of retained teeth in older adults have increased significantly in recent years. It is reported the retention rate of more than 20 natural teeth in the older adult population is $51.25 \%$, and the average retention rate is more than 22.5 teeth in older adults aged 65-74 years (14). Therefore, the retention of the OA is effectively guaranteed, which supplies excellent prospects for its application in older adults' OSAS treatment.

In this study, after treatment with the adjustable OA for 6 months, the subjective symptoms of the older adult patients with OSAS were improved to varying degrees, especially in snoring and suffocating, while it was not apparent in the daytime sleepiness and memory. The reasons for daytime sleepiness in older adult patients with OSAS are not yet clear, but some studies have shown that it is related to sleep disorders and mental and psychological factors (15).

The older adults are prone to severe hypoxia due to airway collapse, central respiratory dysfunction, and the complications of the lungs and the cardiovascular system (16). We found the respiratory disturbance index of older adult patients with OSAS was improved after treatment with the OA, especially in terms of blood oxygen saturation, which indicates the adjustable OA has a positive clinical effect on older adult patients with OSAS.

The etiology of older adult patients with OSAS is closely related to their pathophysiological changes. Anatomical stenosis of the upper airway is the main reason for OSAS, in which the velopharyngeal plane and glossopharyngeal plane stenosis are the most common. The decrease of physiological function, the decrease of respiratory muscle tension, and the increase of soft tissue motility can aggravate the collapsibility while increasing the resistance of the upper airway. Sleep disorders and the increase of light sleep incidences are also the inducements for OSAS $(17,18)$.

Cone-beam CT has significant clinical reference value for the convenient and correct measurement of the threedimensional shape of the upper airway. The results of the CBCT examination in this study showed that the palatal and glossopharyngeal air passages of older adult OSAS patients were significantly enlarged. The efficacy of adjustable OAs for older adult OSAS patients is further verified, and the structural stenosis of the palatal and glossopharyngeal has also been confirmed to be a significant cause of OSAS, which was consistent with a previous analysis result of spiral 
CT and MRI in literature (19).

Only when the clinical symptoms are apparent, older adult OSAS patients will seek medical treatment, which will quickly cause sample deviation. The minor specimen would also affect the experimental results. The long-term efficacy and complications of OAs for older adult OSAS patients still require further follow-up in the clinical practice.

\section{Limitations}

Firstly, this trial was only an observational trial and not a randomized controlled trial. Secondly, this study was only a single-center trial, and the sample size was limited. Thirdly, there was no control group in this study.

\section{Conclusions}

The adjustable OA had extensive clinical efficacy and comfort in older adult OSAS patients by enlarging the palatopharynx and glossopharynx.

\section{Acknowledgments}

Funding: This study was financially supported by Capital Clinical Characteristic Application Research (No. Z171100001017192), Beijing Excellent Talents Training Project (No. 2017000062586G232)and Natural Science Foundation of Beijing (No. 7182125)

\section{Footnote}

Reporting Checklist: The authors have completed the TREND reporting checklist. Available at http://dx.doi. org/10.21037/apm-20-1300

Data Sharing Statement: Available at http://dx.doi. org/10.21037/apm-20-1300

Conflicts of Interest: All authors have completed the ICMJE uniform disclosure form (available at http://dx.doi. org/10.21037/apm-20-1300). The authors have no conflicts of interest to declare.

Ethical Statement: The authors are accountable for all aspects of the work in ensuring that questions related to the accuracy or integrity of any part of the work are appropriately investigated and resolved. All procedures performed in this study involving human participants were in accordance with the Declaration of Helsinki (as revised in 2013). The study was approved by Ethics Committee of the 307 Hospital of PLA (No. ky-2018-9-83). And all patients signed informed consent.

Open Access Statement: This is an Open Access article distributed in accordance with the Creative Commons Attribution-NonCommercial-NoDerivs 4.0 International License (CC BY-NC-ND 4.0), which permits the noncommercial replication and distribution of the article with the strict proviso that no changes or edits are made and the original work is properly cited (including links to both the formal publication through the relevant DOI and the license). See: https://creativecommons.org/licenses/by-nc-nd/4.0/.

\section{References}

1. Destors M, Tamisier R, Galerneau LM, et al. Pathophysiology of obstructive sleep apnea syndrome and its cardiometabolic consequences. Presse Med 2017;46:395-403.

2. Wu M, Zhou L, Zhu D, et al. Hematological indices as simple, inexpensive and practical severity markers of obstructive sleep apnea syndrome: a meta-analysis. J Thorac Dis 2018;10:6509-21.

3. Cossellu G, Biagi R, Sarcina M, et al. Three-dimensional evaluation of upper airway in patients with obstructive sleep apnea syndrome during oral appliance therapy. J Craniofac Surg 2015;26:745-8.

4. Hernández Voth A, Sayas Catalán J, Benavides Mañas P, et al. Obstructive sleep apnea-hypopnea syndrome in patients with severe chronic respiratory insufficiency. Medicina Clínica 2017;148:449-52.

5. You M, Zhang L, Fang L, et al. Evaluation of carotid arterial elasticity in patients with obstructive sleep apnea hypopnea syndrome by two-dimensional speckle tracking imaging. Medicine 2017;96:e8817.

6. Joosten SA, Khoo JK, Edwards BA, et al. Improvement in obstructive sleep apnea with weight loss is dependent on body position during sleep. Sleep 2017;40:332-8.

7. Punchik B, Press Y, Goldbart A. Syndrome of obstructive sleep apnea syndrome in the elderly population-prevalence, clinical characteristics and treatment strategy. Harefuah 2017;156:41-4.

8. Wang S, Niu X, Zhang P, et al. Analysis of OSAS incidence and influential factors in middle-aged and elderly patients with hypertension. Minerva Med 2019;110:115-20.

9. Ifergane G, Ovanyan A, Toledano R, et al. Obstructive 
sleep apnea in acute stroke: a role for systemic inflammation. Stroke 2016,47:1207-12.

10. Crawford-Achour E, Dauphinot V, Martin MS, et al. Protective Effect of Long-Term CPAP Therapy on cognitive performance in elderly patients with severe OSA: the pROOF study. J Clin Sleep Med 2015;11:519-24.

11. Balcan B, Uğurlu AÖ. Results of polysomnographies and treatment strategies in elderly patients with symptoms of obstructive sleep apnea syndrome. Turk Thorac J 2017;18:108-13.

12. Cantore S, Ballini A, Farronato D, et al. Evaluation of an oral appliance in patients with mild to moderate obstructive sleep apnea syndrome intolerant to continuous positive airway pressure use: Preliminary results. Int J Immunopathol Pharmacol 2016;29:267-73.

13. de Lourdes Rabelo Guimarães M, Hermont AP, de Azevedo PG, et al. Severe obstructive sleep apnea treatment with oral appliance: the impact on obstructive, central and mixed events. Sleep Breath 2018,22:91-8.

14. Carra MC, Schmitt A, Thomas F, et al. Sleep disorders

Cite this article as: Lu RJ, Tian N, Wang JZ, Zou X, Wang JJ, Zhang M, Bai CQ, Yu KT. The effectiveness of adjustable oral appliance for older adult patients with obstructive sleep apnea syndrome. Ann Palliat Med 2020;9(4):2178-2186. doi: 10.21037/ apm-20-1300 and oral health: a cross-sectional study. Clin Oral Investig 2017;21:975-83.

15. Ingravallo F, Schenck CH, D'Aloja E, et al. Firing a loaded gun during sleep in an elderly man with a "perfect storm" of risk factors including severe obstructive sleep apnea. Sleep Breath 2018;22:51-4.

16. Mahlberg R. Sleep Disturbances in the Elderly. Dtsch Med Wochenschr 2016;141:1138-40.

17. Boutet C, Abdirahman Mohamed Moussa S, Celle S, et al. Supra-epiglottic upper airway volume in elderly patients with obstructive sleep apnea hypopnea syndrome. PLoS One 2016;11:e0157720.

18. Yetkin O, Aydogan D. Effect of CPAP on sleep spindles in patients with OSA. Respir Physiol Neurobiol 2018;247:71-3.

19. Yilmaz Z, Voyvoda N, Ínan E, et al. Factors affecting executive functions in obstructive sleep apnea syndrome and volumetric changes in the prefrontal cortex. Springerplus 2016;5:1934.

(English Language Editor: J. Chapnick) 